\title{
Construção de um instrumento de triagem do vocabulário para crianças entre 3 e 7 anos
}

\section{Development of a vocabulary-screening tool for children 3 to 7-years of age}

\author{
Alexandre Lucas de Araújo Barbosaํㅜ (1), Heliana Bezerra Soares² (1), Cíntia Alves Salgado Azoni
}

\begin{abstract}
RESUMO
Objetivo: construir um instrumento de triagem do vocabulário para crianças entre 3 e 7 anos de idade. Métodos: a etapa 1, de construção do instrumento, consistiu em revisão da literatura nacional e internacional, para verificar os instrumentos utilizados para triagem do vocabulário, por meio de pesquisa nas bases de dados SciELO, LILACS, ERIC e PubMed. Na etapa 2, denominada formulação de itens e manual de uso, foi elaborado um instrumento direcionado para o vocabulário, com a criação de um banco de dados de palavras para crianças de 3 a 7 anos, de acordo com 11 categorias semânticas, em habilidades expressivas e receptivas. Por fim, a etapa 3 consistiu na criação das fichas de aplicação do instrumento. Resultados: na primeira etapa do estudo, foram identificados três instrumentos internacionais para triagem do vocabulário. Nenhum instrumento nacional foi encontrado. Quanto à segunda etapa, o banco de dados consistiu em um total de 210 palavras, dentre elas, substantivos, adjetivos e verbos. Na terceira etapa, foram criadas as imagens, manual e folhas de resposta. Conclusão: verificou-se escassez de instrumentos de triagem do vocabulário na literatura internacional e nacional. Portanto, foi construído um instrumento de triagem do vocabulário infantil para crianças entre 3 e 7 anos de idade.
\end{abstract}

Palavras-chave: Programas de rastreamento; Protocolos; Linguagem infantil; Testes de vocabulário; Vocabulário

\begin{abstract}
Purpose: To develop a vocabulary screening tool for children from 3 to 7 years of age. Methods: Step one, development of the instrument, consisted of an integrative review of the National and International literature to verify instruments for vocabulary screening, and used the SciELO, LILACS, ERIC and PubMed databases. For step two, the items and user's manual were developed. A vocabulary-oriented instrument was developed: a database of words for children from 3 to 7 years old comprised 11 semantic categories and tapped expressive as well as receptive skills. The final step involved the creation of protocols for the instrument. Results: In the first step of the study, three international vocabulary screening instruments were identified. No national instrument was found. For the second step, a set of a total of 210 words were selected, including nouns, adjectives and verbs. During the third and final step, images, manual and answer protocols were developed. Conclusion: There was a scarcity of vocabulary screening instruments identified in international and national literature. Therefore, a vocabulary screening instrument for children between 3 and 7 years of age was developed.
\end{abstract}

Keywords: Screening tool; Protocols; Child language; Vocabulary test; Vocabulary

\footnotetext{
Trabalho realizado no Departamento de Fonoaudiologia, em parceria com o Departamento de Engenharia Biomédica, Universidade Federal do Rio Grande do Norte - UFRN - Natal (RN), Brasil.

${ }^{1}$ Departamento de Fonoaudiologia, Centro de Ciências da Saúde, Universidade Federal do Rio Grande do Norte - UFRN - Natal (RN), Brasil.

${ }^{2}$ Departamento de Engenharia Biomédica, Universidade Federal do Rio Grande do Norte - UFRN - Natal (RN), Brasil.

Conflito de interesses: Não.

Contribuição dos autores: ALAB, HBS e CASA participaram da concepção e delineamento do estudo, coleta, análise e interpretação dos resultados; ALAB e CASA participaram da redação e revisão do artigo de forma intelectualmente importante.

Financiamento: Bolsa de mestrado concedida pela Coordenação de Aperfeiçoamento de Pessoal de Nível Superior (Capes), processo $\mathrm{n}^{\circ} 1728008$.

Autor correspondente: Alexandre Lucas de Araújo Barbosa. E-mail: ilucasbarbosa@icloud.com

Recebido: Janeiro 17, 2019; Aceito: Setembro 02, 2019.
} 


\section{INTRODUÇÃO}

Atrasos no desenvolvimento de linguagem são frequentemente negligenciados. A abordagem do "esperar para ver"(1) supõe que falantes tardios irão se aproximar de seus pares típicos. Entretanto, os resultados têm sido insatisfatórios, pois muitas crianças apresentam problemas persistentes. Ainda, aquelas que superam o atraso continuam apresentando desempenho linguístico inferior, quando comparadas às crianças sem esse histórico ${ }^{(2)}$.

A dificuldade na aquisição do vocabulário é um dos principais indicadores de alterações na linguagem oral ${ }^{(3)}$, podendo acarretar prejuízos no processo de aprendizagem da leitura $^{(4)}$, na compreensão leitora ${ }^{(5)}$, problemas emocionais e comportamentais $^{(6)}$.

Um dos meios mais adotados, mundialmente, na área da saúde, para a detecção precoce de agravos é a triagem. Por definição, é um procedimento para identificar indivíduos em risco para determinada condição ${ }^{(7)}$. Levando em consideração a importância do vocabulário, a triagem desta habilidade possibilita a identificação de crianças com dificuldades, ou possíveis atrasos ${ }^{(8)}$.

A detecção e a intervenção precoce garantem melhor prognóstico infantil e aprimoramento de habilidades requeridas ao processo escolar ${ }^{(9)}$. Todavia, ainda é escasso o número de pesquisas envolvendo a triagem do vocabulário ${ }^{(8)}$, gerando, como consequência, a identificação tardia de crianças com possíveis alterações nesse nível da linguagem.

Tendo em vista a importância do vocabulário e da detecção de dificuldades em seu desenvolvimento, o objetivo desta pesquisa foi construir um instrumento de triagem do vocabulário para crianças entre 3 e 7 anos de idade, em sua versão manual.

\section{MÉTODO}

\section{Aspectos éticos}

Esta pesquisa foi aprovada pelo Comitê de Ética em Pesquisa (CEP) da Universidade Federal do Rio Grande do Norte - UFRN, sob parecer $\mathrm{n}^{\mathrm{O}} 2.548 .341$, seguindo a resolução do Conselho Nacional de Saúde - CNS 466/12 sobre Diretrizes e Normas Regulamentadoras de Pesquisas Envolvendo Seres Humanos.

Este estudo baseou-se em dados teóricos e, portanto, a assinatura do Termo de Consentimento Livre e Esclarecido (TCLE) foi dispensada pelo CEP.

\section{Etapas da pesquisa}

Trata-se de um estudo descritivo e qualitativo, que abrange a descrição dos processos de construção teórica, definição dos objetivos do instrumento, seleção dos itens e forma de aplicação. A construção do instrumento em questão, nomeado TRILHAR, ocorreu em três etapas: 1) revisão da literatura; 2) formulação dos itens e do manual de uso; 3) criação das fichas de aplicação.

\section{Etapa 1: revisão da literatura}

\section{Estratégia de pesquisa}

Inicialmente, foi realizada uma revisão integrativa da literatura, para verificar os instrumentos de triagem do vocabulário disponíveis nacionalmente e internacionalmente, por meio das bases de dados Scientific Electronic Library Online (SciELO), Literatura Latino-Americana e do Caribe em Ciências da Saúde (LILACS), Education Resources Information Center (ERIC) e PubMed.

As buscas nas bases de dados nacionais SciELO e LILACS foram realizadas a partir dos Descritores em Ciências da Saúde (DeCS), utilizando as seguintes combinações: triagem $A N D$ linguagem infantil, triagem $A N D$ vocabulário, triagem $A N D$ testes de vocabulário, programas de rastreamento $A N D$ linguagem infantil, programas de rastreamento $A N D$ vocabulário, programas de rastreamento $A N D$ testes de vocabulário, linguagem infantil $A N D$ vocabulário, linguagem infantil $A N D$ teste de vocabulário, vocabulário $A N D$ teste de vocabulário.

Para a pesquisa na ERIC, as seguintes combinações de descritores da própria base foram utilizadas: screening tests AND vocabulary, screening tests AND child language, screening tests $A N D$ vocabulary $A N D$ child language. Por fim, para a PubMed, foram utilizados descritores da Medical Subject Headings (MeSH): mass screening AND child language, mass screening AND vocabulary, mass screening AND language tests, language tests AND vocabulary.

\section{Critérios de seleção}

Foram considerados artigos de acordo com os seguintes critérios de inclusão: artigos que utilizaram instrumentos de triagem do vocabulário, disponíveis na íntegra, publicados em português ou inglês, entre janeiro de 2014 e dezembro de 2018 . Foram excluídos os artigos que não abordaram o assunto no título ou resumo, que não indicaram o nome do instrumento, estudos repetidos entre as plataformas e de revisão de literatura.

A seleção dos estudos foi realizada conforme a leitura do título e resumo. Posteriormente, os artigos que atenderam aos critérios foram lidos na íntegra, para a coleta das informações.

\section{Análise dos dados}

A análise dos dados coletados nos artigos foi realizada de acordo com o protocolo, incluindo as seguintes informações: nome do instrumento, nacionalidade, faixa etária, formato e estudos relacionados, ou seja, estudos que utilizaram o mesmo instrumento. Por fim, estas informações foram incluídas em tabela, analisadas e discutidas de acordo com literatura atual (Tabela 1).

\section{Etapa 2: formulação dos itens}

Por se tratar de um instrumento de triagem a ser utilizado no ambiente educacional, inicialmente, os pesquisadores optaram pela faixa etária de 3 a 7 anos, como público-alvo. 
A escolha da abrangência de início aos 3 anos ocorreu em decorrência de condições de aplicabilidade para a educação infantil, considerando que o ingresso na escola ocorre aos 4 anos, porém, algumas crianças podem iniciar um pouco antes, aos 3 anos $^{(13)}$. Quanto à idade máxima de 7 anos, considerou-se o processo da alfabetização no ensino fundamental, momento de aprendizagem da leitura e, portanto, o vocabulário se faz importante na relação com o desenvolvimento da consciência fonológica $^{(14)}$ e compreensão da leitura ${ }^{(10)}$.

A formulação dos itens para cada faixa etária foi baseada em dois instrumentos nacionais, que têm, como objetivo, a avaliação do vocabulário expressivo e receptivo: o ABFW - Teste de Linguagem Infantil ${ }^{(15)} \mathrm{e}$ o Teste de Vocabulário por Figuras USP - TVfUSP(11), respectivamente. Assim, a construção do banco de palavras em tabela, quanto às habilidades receptivas e expressivas, foi realizada levando em consideração os seguintes critérios de inclusão: ter o mínimo possível de variação cultural; ser de fácil representação gráfica e pertencer às categorias semânticas de móveis, brinquedos, partes do corpo, roupas e calçados, animais, meios de transporte, comidas, locais, profissões, verbos e adjetivos. Neste momento, não foi estipulada uma quantidade de palavras a ser selecionada, por se tratar apenas de uma seleção inicial das possíveis palavras que poderiam compor a triagem. A escolha se embasou em dois testes nacionais de avaliação em vocabulário, bem como na experiência clínica de identificar possíveis palavras comuns de utilização no ambiente escolar para essa faixa etária, que não sofressem variações regionais significativas. No entanto, é importante ressaltar que, após a etapa de validação do instrumento, as palavras poderão ser confirmadas como adequadas, ou não, para a faixa etária, mediante análise de juízes da área e aplicabilidade do instrumento em crianças de diferentes contextos sociais e culturais.

Após a etapa de construção do banco de palavras, foram selecionados dez vocábulos para compor as atividades de vocabulário receptivo e dez para compor as atividades de vocabulário expressivo, para cada faixa etária (Quadro 1). A divisão de acordo com as modalidades expressiva e receptiva foi realizada levando em consideração os protocolos de base. Desta forma, as palavras pertencentes ao ABFW - Teste de Linguagem Infantil foram selecionadas para o vocabulário expressivo e as palavras do TVfUSP, para o vocabulário receptivo, visto que ambos os protocolos já foram submetidos a estudos com a população brasileira. Além disso, para o vocabulário expressivo, foram selecionadas palavras de duas a três sílabas para as crianças até 5 anos, enquanto para as crianças de 6 e 7 anos, foram utilizadas palavras de maior extensão silábica, levando em conta que, nesta idade, o desenvolvimento fonológico já foi finalizado. Para o vocabulário receptivo, não foram utilizados estes critérios, pois não há necessidade de produção da criança durante a atividade.

Já a divisão para a faixa etária, visto que não existem estudos brasileiros que fundamentem esta escolha, foi utilizada, como critério, a experiência dos pesquisadores na área de linguagem e educação. Sendo assim, o cuidado dos pesquisadores foi direcionado à escolha de palavras com representações lexicais minimamente comuns para as diferentes regiões e contextos culturais do Brasil.

\section{Etapa 3: criação das fichas de aplicação, do manual de uso e da folha de marcação}

No primeiro momento, a etapa de criação das fichas de aplicação do instrumento em sua versão manual foi realizada a partir da construção de imagens correspondentes às palavras selecionadas, por meio da contratação de um designer com experiência em figuras infantis. Após, as figuras foram inseridas nas fichas de atividades de vocabulário receptivo e expressivo, utilizando o Adobe ${ }^{\circledR}$ Photoshop CC.

As fichas de aplicação do vocabulário expressivo foram compostas por uma figura cada, visando a nomeação de um único estímulo por vez. Já as fichas do vocabulário receptivo, foram compostas por quatro imagens, sendo uma o estímulo-alvo a ser identificado pela criança, e as outras três, figuras distratoras, compostas de imagens já pertencentes ao

Tabela 1. Descrição dos protocolos utilizados na literatura estudada

\begin{tabular}{|c|c|c|c|c|}
\hline INSTRUMENTO & NACIONALIDADE & FAIXA ETÁRIA & FORMATO & ESTUDOS RELACIONADOS \\
\hline $\begin{array}{l}\text { Dynamic Indicators of } \\
\text { Vocabulary Skills (DIVS) }\end{array}$ & Estados Unidos & 4 anos de idade & Triagem direta & $\begin{array}{l}\text { Marcotte, Parker, Furey, Hands }{ }^{(8)} \\
\text { Marcotte, Clemens, Parker, Whitcomb(10) }\end{array}$ \\
\hline $\begin{array}{l}\text { Language Development } \\
\text { Survey (LDS) }\end{array}$ & Estados Unidos & 2 anos de idade & $\begin{array}{l}\text { Questionário para os } \\
\text { pais }\end{array}$ & Rescola, Nyame, Dias ${ }^{(11)}$ \\
\hline $\begin{array}{l}\text { Receptive Vocabulary } \\
\text { Screener Aplication (RVS) }\end{array}$ & Reino Unido & $\begin{array}{l}3 ; 6 \text { a } 6 \text { anos de } \\
\text { idade }\end{array}$ & $\begin{array}{l}\text { Triagem direta via } \\
\text { aplicativo eletrônico }\end{array}$ & $\begin{array}{l}\text { Schaefer, Bowyer-Crane, Herrmann, } \\
\text { Fricke }^{(12)}\end{array}$ \\
\hline
\end{tabular}

Quadro 1. Palavras selecionadas para a triagem de cada faixa etária

\begin{tabular}{|c|c|c|c|c|c|}
\hline & 3 ANOS & 4 ANOS & 5 ANOS & 6 ANOS & 7 ANOS \\
\hline $\begin{array}{l}\text { Vocabulário } \\
\text { receptivo }\end{array}$ & $\begin{array}{c}\text { Morder, andar, } \\
\text { geladeira, abacaxi, } \\
\text { laranja, casa, maçã, } \\
\text { janela, pular, vaca }\end{array}$ & $\begin{array}{l}\text { Correr, igreja, dormir, } \\
\text { avião, formiga, } \\
\text { bicicleta, sapato, } \\
\text { chorar, pão, televisão }\end{array}$ & $\begin{array}{c}\text { Cortar, tambor, } \\
\text { amassar, lixeiro, } \\
\text { vestido, baleia, xícara, } \\
\text { chinelo, lamber, } \\
\text { montanha }\end{array}$ & $\begin{array}{c}\text { Beber, sanduíche, } \\
\text { bater, escova de } \\
\text { dentes, pentear, coruja, } \\
\text { girafa, latir, bombeiro, } \\
\text { balanço }\end{array}$ & $\begin{array}{c}\text { Dominó, telefonar, } \\
\text { meia, porco, pescar, } \\
\text { cantar, cérebro, } \\
\text { ambulância, cozinheiro, } \\
\text { garfo }\end{array}$ \\
\hline $\begin{array}{l}\text { Vocabulário } \\
\text { expressivo }\end{array}$ & $\begin{array}{l}\text { Pato, bolo, fogão, } \\
\text { colher, gangorra, } \\
\text { escola, rir, cenoura, } \\
\text { galinha, torneira }\end{array}$ & $\begin{array}{l}\text { Camisa, cavalo, } \\
\text { banana, mesa, carro, } \\
\text { boneca, palhaço, } \\
\text { quarto, boca, quebrar }\end{array}$ & $\begin{array}{c}\text { Casaco, macaco, } \\
\text { pipoca, relógio, ônibus, } \\
\text { robô, médico, banheiro, } \\
\text { nariz, sentar }\end{array}$ & $\begin{array}{l}\text { Bolsa, elefante, pastel, } \\
\text { toalha, foguete, roda- } \\
\text { gigante, fazendeiro, } \\
\text { praia, umbigo, balançar }\end{array}$ & $\begin{array}{c}\text { Jacaré, queijo, } \\
\text { vassoura, helicóptero, } \\
\text { apito, astronauta, } \\
\text { castelo, aranha, cavar, } \\
\text { dirigir }\end{array}$ \\
\hline
\end{tabular}


banco de dados. A seleção das palavras para cada atividade (receptivo e expressivo) ocorreu aleatoriamente, visto que todas as palavras foram consideradas de fácil identificação da criança por faixa etária.

O manual de uso e a folha de marcação foram produzidos com o objetivo de orientar o aplicador da triagem, contendo tópicos, como indicações, habilidades avaliadas, material, procedimento de aplicação, análise dos resultados e conduta.

\section{RESULTADOS}

\section{Etapa 1}

A busca inicial nas bases de dados resultou em um total de 1199 artigos e, após a leitura dos títulos, 29 foram selecionados para a análise dos resumos. Depois da leitura dos resumos, 13 estudos foram lidos na íntegra, dos quais, 5 foram excluídos por repetição e 4 por não atenderem aos critérios de inclusão estabelecidos. A amostra final foi composta por 4 artigos (Figura 1).

Dos 4 artigos analisados, foi observada a utilização de 3 instrumentos internacionais de triagem do vocabulário, dos quais, 1 foi utilizado em 2 artigos, listados na Tabela 1. Não foram encontrados instrumentos nacionais com este objetivo.

\section{Dynamic Indicators of Vocabulary Skills (DIVS)}

O DIVS realiza triagem do vocabulário receptivo e expressivo de crianças pré-escolares. Seu primeiro subteste, picture naming fluency (PNF), é composto por 44 figuras que devem ser nomeadas em um tempo de um minuto. No segundo

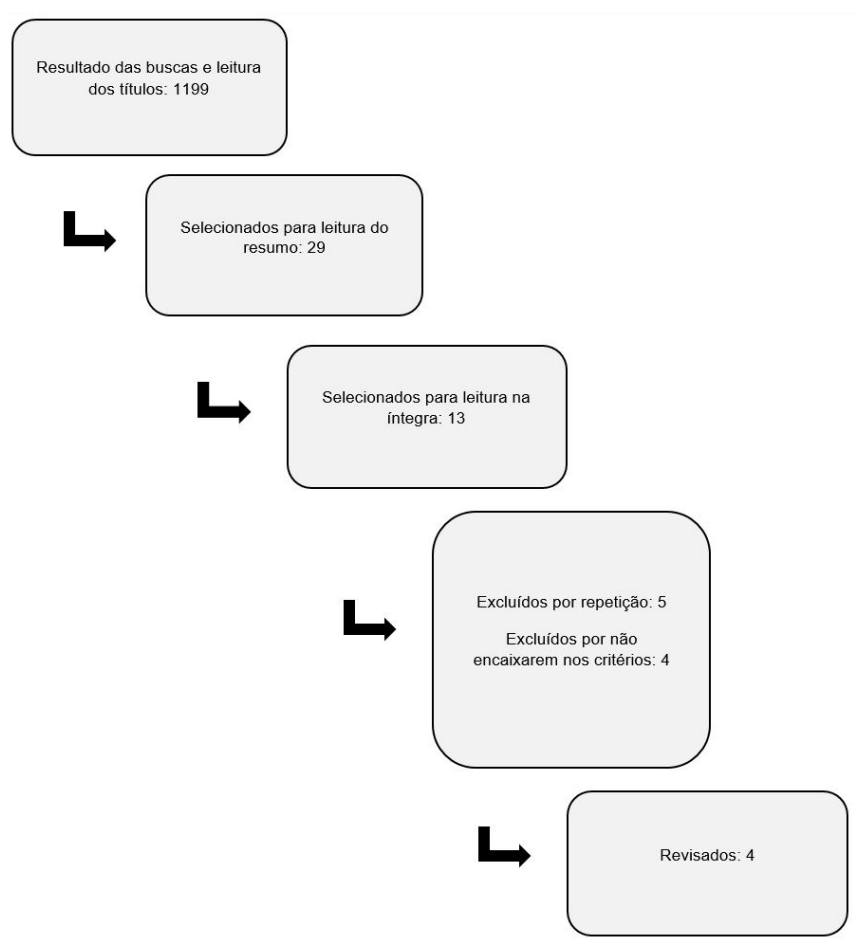

Figura 1. Fluxograma de seleção dos artigos subteste, reverse definition fluency (RDF), o aplicador descreve 30 palavras, as quais a criança deve nomear. A pontuação final do instrumento é composta pelo número de palavras nomeadas corretamente, em ambos os subtestes ${ }^{(8,12)}$.

\section{Language Development Survey (LDS)}

A Language Development Survey é uma escala a ser preenchida pelo responsável, composta por 300 palavras para caracterizar o vocabulário espontâneo de crianças aos 24 meses de idade ${ }^{(16)}$. No estudo em questão, a versão em português europeu do instrumento foi utilizada ${ }^{(17)}$.

\section{Receptive Vocabulary Screener Application (RVSA)}

Este instrumento é um aplicativo para tablet, que visa à triagem do vocabulário receptivo de crianças monolíngues e bilíngues. Inicialmente, um cadastro com os dados do paciente deve ser preenchido, incluindo nome, data de nascimento e línguas faladas. Em cada tela, a criança ouve uma palavra e deve escolher a figura correspondente, entre quatro opções. $\mathrm{O}$ instrumento ainda possui um mascote digital chamado Meemo, que orienta o processo de aplicação em sua língua materna. A pontuação é calculada automaticamente e transferida para uma planilha em Excel ${ }^{(18)}$.

\section{Etapa 2}

O banco de palavras construído obteve um total de 210 itens, distribuídos entre as idades, como exposto na Tabela 2.

\section{Etapa 3}

No total, a partir do banco de palavras, foram elaboradas 210 fichas, sendo 2 atividades de teste, 10 fichas de vocabulário receptivo e 10 de vocabulário expressivo, para cada idade. Desta forma, inicialmente, cada criança deve responder às atividades apenas da sua idade, porém, nas etapas de validade do instrumento serão aplicadas por completo, para que sejam obtidos dados de base para a aplicação da triagem. Quanto à aplicação do instrumento, na habilidade de vocabulário receptivo, a criança irá ouvir uma palavra e deverá apontar, entre 4 opções, a figura correspondente. Uma das figuras é o estímulo-alvo e as outras três, estímulos distratores. Já no vocabulário expressivo, o objetivo é a nomeação de uma figura por ficha. Em ambos, há a nomeação do estímulo visual sem restrição de tempo. A escolha destes procedimentos para a triagem do vocabulário ocorreu em razão da simplicidade das tarefas, visando tanto à facilitação da aplicação pelo aplicador, quanto à compreensão das ordens, pela criança. Também se optou pela triagem direta e individual, considerando que, em diferentes condições sociais, a participação dos pais pela triagem indireta pode inviabilizar a aplicação na escola e identificação de qualquer sinal indicativo de alterações de linguagem, assim como não é possível analisar o real desempenho da criança em condições coletivas.

O manual foi produzido com o objetivo de instruir o avaliador sobre o funcionamento do instrumento. Neste, encontra-se o 
Tabela 2. Banco de palavras para seleção das atividades de cada faixa etária

\begin{tabular}{|c|c|c|c|c|c|}
\hline CATEGORIAS & 3 ANOS & 4 ANOS & 5 ANOS & 6 ANOS & 7 ANOS \\
\hline Móveis & $\begin{array}{l}\text { Colher, janela, } \\
\text { torneira, geladeira, } \\
\text { fogão, copo }\end{array}$ & $\begin{array}{l}\text { Cama, mesa, cadeira, } \\
\text { pia, televisão, faca }\end{array}$ & $\begin{array}{l}\text { Xícara, relógio, pente, } \\
\text { prato, panela }\end{array}$ & $\begin{array}{l}\text { Espelho, toalha, } \\
\text { ventilador, escova } \\
\text { dental, computador }\end{array}$ & $\begin{array}{l}\text { Sofá, vela, roda } \\
\text { (de carro), ferro de } \\
\text { passar, telefone, } \\
\text { vassoura, frigideira, } \\
\text { garfo }\end{array}$ \\
\hline Brinquedos & Gangorra & Boneca, bola, avião & Robô, tambor & $\begin{array}{l}\text { Balanço, roda- } \\
\text { gigante, piano, patins }\end{array}$ & Flecha, apito, dominó \\
\hline Partes do corpo & & Boca, olho, pé & $\begin{array}{l}\text { Perna, nariz, barriga, } \\
\text { dente, orelha }\end{array}$ & $\begin{array}{l}\text { Joelho, dedo, ombro, } \\
\text { umbigo }\end{array}$ & Coração, cérebro \\
\hline Roupas e calçados & & Camisa, sapato & $\begin{array}{l}\text { Calça, chinelo, } \\
\text { vestido, casaco }\end{array}$ & $\begin{array}{l}\text { Chapéu, vestido, } \\
\text { bolsa }\end{array}$ & Meia \\
\hline Animais & $\begin{array}{l}\text { Cachorro, vaca, } \\
\text { galinha, pato }\end{array}$ & $\begin{array}{l}\text { Gato, peixe, sapo, } \\
\text { pássaro, formiga, } \\
\text { rato, cavalo }\end{array}$ & $\begin{array}{l}\text { Macaco, leão, urso, } \\
\text { baleia }\end{array}$ & $\begin{array}{l}\text { Tubarão, elefante, } \\
\text { cobra, coruja, girafa }\end{array}$ & $\begin{array}{l}\text { Baleia, abelha, porco, } \\
\text { coelho, aranha, jacaré }\end{array}$ \\
\hline Meios de transporte & & Carro, bicicleta & $\begin{array}{l}\text { Moto, avião, trem, } \\
\text { ônibus }\end{array}$ & $\begin{array}{l}\text { Caminhão, foguete, } \\
\text { balão }\end{array}$ & $\begin{array}{l}\text { Ambulância, } \\
\text { helicóptero }\end{array}$ \\
\hline Comidas & $\begin{array}{l}\text { Abacaxi, cenoura, } \\
\text { laranja, maçãa, bolo }\end{array}$ & $\begin{array}{l}\text { Banana, pão, sorvete, } \\
\text { ovo }\end{array}$ & $\begin{array}{l}\text { Refrigerante, } \\
\text { morango, pipoca }\end{array}$ & $\begin{array}{l}\text { Melancia, sanduíche, } \\
\text { pastel }\end{array}$ & $\begin{array}{l}\text { Queijo, macarrão, } \\
\text { cebola, uva }\end{array}$ \\
\hline Locais & Casa, escola & Quarto, sala, igreja & $\begin{array}{l}\text { Banheiro, montanha, } \\
\text { parque }\end{array}$ & $\begin{array}{l}\text { Sala de aula, loja, } \\
\text { praia }\end{array}$ & Castelo, rio, cachoeira \\
\hline Profissões & & Professora, palhaço & $\begin{array}{l}\text { Médico, dentista, } \\
\text { lixeiro }\end{array}$ & $\begin{array}{l}\text { Fazendeiro, } \\
\text { bombeiro, pedreiro }\end{array}$ & $\begin{array}{l}\text { Carteiro, cozinheiro, } \\
\text { astronauta }\end{array}$ \\
\hline Verbos & Pular, andar, morder, rir & $\begin{array}{l}\text { Comer, correr, } \\
\text { quebrar, dormir, } \\
\text { chorar }\end{array}$ & $\begin{array}{l}\text { Pentear, amassar, } \\
\text { queimar, lamber, } \\
\text { sentar, cortar }\end{array}$ & $\begin{array}{l}\text { Bater, apagar, } \\
\text { balançar, desenhar, } \\
\text { beber, cheirar, latir }\end{array}$ & $\begin{array}{l}\text { Molhar, cavar, pescar, } \\
\text { telefonar, escrever, } \\
\text { voar, cantar, dirigir, } \\
\text { dançar }\end{array}$ \\
\hline Adjetivos & $\begin{array}{l}\text { Triste, feliz, alto, } \\
\text { grande }\end{array}$ & $\begin{array}{l}\text { Velho, quente, triste, } \\
\text { magro, bravo }\end{array}$ & $\begin{array}{l}\text { pequeno, doente, } \\
\text { gelado, rosa, } \\
\text { quebrado, sentado, } \\
\text { aberto }\end{array}$ & $\begin{array}{l}\text { fechado, zangado, } \\
\text { redondo, ensolarado, } \\
\text { sujo, grávida, } \\
\text { Cacheado }\end{array}$ & $\begin{array}{l}\text { Forte, moreno, } \\
\text { barbudo, preso, } \\
\text { florido, curto, vazio, } \\
\text { fundo }\end{array}$ \\
\hline
\end{tabular}

texto de fundamentação teórica sobre o desenvolvimento típico e atípico do vocabulário e a importância da detecção precoce de alterações. Além disso, são listados os materiais que compõem o instrumento (fichas de triagem do vocabulário expressivo e receptivo, um fantoche e folhas de resposta), os procedimentos de aplicação, a forma de preenchimento da folha de resposta e análise dos dados.

A triagem deve ser realizada em um local com condições acústicas e de iluminação adequadas, com crianças entre 3 e 7 anos de idade. $\mathrm{O}$ material inclui um fantoche de um cachorro como mascote, para facilitar a interação entre aplicador e criança e deixá-la mais à vontade para o procedimento, criando um ambiente lúdico e interativo para a observação de aspectos qualitativos.

A folha de respostas permitirá a análise e registro do desempenho da criança. Cada item deve ser pontuado em (1) para acerto e (0) para erro, com um total máximo de 20 pontos. Embora o objetivo seja o vocabulário, sugere-se, também, a observação de outros aspectos qualitativos da linguagem, como a presença de alterações fonológicas na oralidade e dificuldades na compreensão das ordens; e de comportamento, como desatenção e agitação.

Por fim, após a aplicação, será gerado um relatório para entrega aos professores e pais para encaminhamento, caso necessário. O profissional fonoaudiólogo deverá tomar as medidas, de acordo com cada paciente.

\section{DISCUSSÃO}

O objetivo deste trabalho foi construir um instrumento de triagem do vocabulário receptivo e expressivo para crianças entre 3 e 7 anos de idade. A primeira etapa, constituída da revisão integrativa de literatura, resultou em três instrumentos de triagem do vocabulário, disponíveis em outros países.

O primeiro, o Dynamic Indicators of Vocabulary Skills foi submetido ao processo de validação, apresentando valores de confiabilidade, validade de construto e validade preditiva adequadas ${ }^{(12)}$. O desempenho, nesta triagem, prediz o desenvolvimento do vocabulário receptivo e expressivo, importantes para a aquisição da leitura e sucesso acadêmico ${ }^{(19)}$.

O segundo artigo descreveu o Language Development Survey, instrumento elaborado como um checklist traduzido para diversos idiomas. Suas evidências de validade confirmam a capacidade de diferenciação de crianças com atrasos no desenvolvimento da linguagem ${ }^{(20)}$. Apesar disso, é possível que os resultados, nesta triagem, possam ser influenciados pelo nível socioeconômico e educacional dos pais, comprometendo a fidedignidade do instrumento $^{(21)}$. Desta forma, para sua aplicação no Brasil, é essencial o processo de adaptação transcultural e validação.

Por fim, apesar da ausência de dados de validação, o Receptive Vocabulary Screener Application, descrito no quarto artigo, apresentou adequada usabilidade para a triagem de crianças monolíngues e bilíngues. Este aplicativo pode ser 
utilizado por profissionais da área da saúde e educação(18). Sua utilização em tablet acompanha os avanços tecnológicos, visto que grande parte das crianças são familiarizadas com este tipo de aparelho ${ }^{(22)}$.

Em relação ao cenário nacional, não foram encontrados instrumentos de triagem do vocabulário. Este dado reforça a necessidade da criação e validação de instrumentos nacionais na área da Fonoaudiologia, visto que o número de estudos dedicados a esse aspecto ainda é reduzido ${ }^{(23)}$. Como resultado, há um número escasso de testes fonoaudiológicos comercialmente disponíveis ${ }^{(24)}$.

Além de demonstrar a escassez de instrumentos de triagem do vocabulário no cenário nacional, os artigos revisados contribuíram como fonte de pesquisa para as possibilidades de aplicação do instrumento. A triagem direta foi escolhida como forma de aplicação, pois as respostas para uma triagem indireta por meio de questionários e checklist pode ser influenciada pelas condições socioeconômicas e educacionais da família em questão ${ }^{(21)}$. Outro aspecto é que, apesar da utilização de ferramentas tecnológicas apresentarem otimização e motivação na aplicação para as crianças, é importante destacar que, em muitos contextos educacionais brasileiros, esta pode ser uma barreira na triagem por parte do profissional, em razão da dificuldade de acesso a tecnologias no ambiente profissional.

Desta forma, a revisão evidenciou a importância de monitorar o desenvolvimento do vocabulário, inclusive pela importante relação para o desenvolvimento de habilidades posteriores, como a decodificação e compreensão leitora ${ }^{(19,25)}$, bem como as formas de uso dos instrumentos para elaboração desta triagem em questão. Com isso, as habilidades de vocabulário receptivo e expressivo foram escolhidas para compor o instrumento, pois a produção de poucas palavras pode ser um dos principais indicadores de atrasos no desenvolvimento da linguagem ${ }^{(19)}$, característica que pode ser observada em diagnósticos, como no transtorno do desenvolvimento da linguagem (TDL) ${ }^{(26)}$, transtorno do espectro do autismo ${ }^{(27)}$ e atraso de linguagem ${ }^{(28)}$.

Espera-se que este instrumento seja capaz de diferenciar o vocabulário de crianças que possuam alterações no desenvolvimento da linguagem, especificamente no vocabulário, possibilitando a identificação e intervenção precoces, que acarretarão benefícios importantes no prognóstico infantil. O instrumento aqui exposto poderá ser modificado de acordo com as futuras etapas de validação, para adequação de aspectos indicados por juízes avaliadores, ou observados nos estudos de aplicação com as crianças, como, por exemplo, a ampliação da faixa etária e o uso de palavras apropriadas, conforme variantes linguísticas de diferentes regiões.

Para verificar, entretanto, se os resultados do instrumento são confiáveis, são necessárias pesquisas de validação, que incluem processos como: validade baseada no conteúdo do teste; validade baseada nos processos de resposta; evidência de validade baseada na consistência interna; confiabilidade; equidade e acurácia ${ }^{(29)}$. Assim, os próximos passos desta pesquisa devem verificar as evidências de validade baseadas no conteúdo do teste. Este dado estima se os itens do instrumento estão de acordo com o propósito do construto, isto é, se os itens selecionados são representativos para a identificação de crianças com alterações no vocabulário ${ }^{(30)}$.

\section{CONCLUSÃO}

Verificou-se a ausência de instrumentos nacionais de triagem do vocabulário. Com base na revisão da literatura, foi construído um instrumento de triagem de vocabulário expressivo e receptivo para crianças entre 3 e 7 anos de idade.

Este estudo pode contribuir com o cenário científico, especialmente no campo da Fonoaudiologia Educacional e da Linguagem, visto a escassez de instrumentos de triagem e a necessidade prática do uso deste tipo de ferramenta em unidades de saúde, escolas e consultórios. A utilização de instrumentos de triagem do vocabulário no ambiente escolar pode trazer benefícios por meio da identificação de crianças de risco para as alterações de linguagem.

\section{AGRADECIMENTOS}

À Coordenação de Aperfeiçoamento de Pessoal de Nível Superior (Capes) pelo apoio para a realização desta pesquisa.

\section{REFERÊNCIAS}

1. Singleton NC. Late talkers: why the wait-and-see approach is outdated. Pediatr Clin North Am. 2018;65(1):13-29. http://dx.doi.org/10.1016/j. pcl.2017.08.018. PMid:29173713.

2. Rescorla L. Age 17 language and reading outcomes in late-talking toddlers: support for a dimensional perspective on language delay. $\mathrm{J}$ Speech Lang Hear Res. 2009;52(1):16-30. http://dx.doi.org/10.1044/10924388(2008/07-0171). PMid:18723598.

3. Gatt D, Grech H, Dodd B. Early lexical expression. In tipically developing Maltese children: implications for the identification of language delay. Clin Linguist Phon. 2013;27(6-7):459-71. http:// dx.doi.org/10.3109/02699206.2013.771213. PMid:23621436.

4. Psyridou M, Eklund K, Poikkeus AM, Torppa M. Reading outcomes of children with delayed early vocabulary: a follow-up from age 2-16. Res Dev Disabil. 2018;78:114-24. http://dx.doi.org/10.1016/j. ridd.2018.05.004. PMid:29805034.

5. Lau KL. Language skills in classical chinese text comprehension. J Psycholinguist Res. 2018;47(1):139-57. http://dx.doi.org/10.1007/ s10936-017-9520-0. PMid:28879414.

6. Henrichs J, Rescorla L, Donkersloot C, Schenk JJ, Raat H, Jaddoe VW, et al. Early vocabulary delay and behavioral/emotional problems in early childhood: the generation R study. J Speech Lang Hear Res. 2013;56(2):553-66. http://dx.doi.org/10.1044/1092-4388(2012/110169). PMid:22896048.

7. Wald NJ. Guidance on terminology. J Med Screen. 2006;13(1):53. http://dx.doi.org/10.1258/096914106776179818. PMid:16569307.

8. Marcotte A, Clemens N, Parker C, Whitcomb S. Examining the classification accuracy of a vocabulary screening measure with preschool children. Assess Eff Interv. 2016;41(4):230-42. http://dx.doi. org/10.1177/1534508416632236.

9. Fricke S, Bowyer-Crane C, Haley AJ, Hulme C, Snowling MJ. Efficacy of language intervention in the early years. J Child Psychol Psychiatry. 2013;54(3):280-90. http://dx.doi.org/10.1111/jcpp.12010. PMid:23176547. 
10. Lau LK. Language skills in classical chinese text comprehension. J Psycholinguist Res. 2018;47(1):139-57. http://dx.doi.org/10.1007/ s10936-017-9520-0. PMid:28879414.

11. Capovilla F. Teste de vocabulário por figuras USP - TVFUSP. $1^{\mathrm{a}}$ ed. São Paulo: Memnon; 2011. 530 p.

12. Marcotte A, Parker C, Furey W, Hands J. An examination of the validity of the dynamic indicators of vocabulary skills (DIVS). J Psychoed Assess. 2014;32(2):133-45. http://dx.doi.org/10.1177/0734282913498849.

13. Brasil. Ministério da Educação. Resolução $n^{\circ} 2$, de 9 de outubro de 2018 [Internet]. Brasília: Ministério da Educação; 2019 [cited 2019 Jun 2]. Available from: http://portal.mec.gov.br/index. php?option $=$ com_docman\&view $=$ download\&alias $=9831$ 1-rceb00218\&category_slug $=$ outubro-2018-pdf-1\&Itemid $=30192$

14. Storch SA, Whitehurst GJ. Oral language and code-related precursors to reading: evidence from a longitudinal structural model. Dev Psychol. 2002;38(6):934-47. http://dx.doi.org/10.1037/0012-1649.38.6.934 PMid:12428705.

15. Andrade C, Befi-Lopes D, Fernandes F, Wertzner H. ABFW: teste de linguagem infantil nas áreas de fonologia, vocabulário, fluência e pragmática. $2^{\mathrm{a}}$ ed. São Paulo: Pró-Fono; 2004. 98 p.

16. Rescorla L. The language development survey: a screening tool for delayed language in toddlers. J Speech Hear Disord. 1989;54(4):58799. http://dx.doi.org/10.1044/jshd.5404.587. PMid:2811339.

17. Rescorla L, Nyame J, Dias P. Vocabulary development in european portuguese: a replication study using the language development survey. J Speech Lang Hear Res. 2016;59(6):1484-90. http://dx.doi. org/10.1044/2016_JSLHR-L-15-0294. PMid:27960004.

18. Schaefer B, Bowyer-Crane C, Herrmann F, Fricke S. Development of a tablet application for the screening of receptive vocabulary skills in multilingual children: a pilot study. Child Lang Teach Ther. 2015;32(2):179-91. http://dx.doi.org/10.1177/0265659015591634.

19. Duff FJ, Reen G, Plunkett K, Nation K. Do infant vocabulary skills predict schoolage language and literacy outcomes. J Child Psychol Psychiatry. 2015;56(8):848-56. http://dx.doi.org/10.1111/jcpp.12378. PMid:25557322.
20. Rescorla L, Alley A. Validation of the language development survey (LDS): a parent report tool for identifying language delay in toddlers. J Speech Lang Hear Res. 2001 Abr;44(2):434-45. http://dx.doi. org/10.1044/1092-4388(2001/035). PMid:11324663.

21. Molini-Avejonas DR. Early identification of language delay by direct language assessment or parent report. Rev Soc Bras Fonoaudiol. 2012;17(4):504. http://dx.doi.org/10.1590/S1516-80342012000400024.

22. Geist E. Toddlers through preschool: using tablet computers with toddlers and young preschoolers. YC Young Child. 2014;69(1):58-63.

23. Gurgel L, Kaiser V, Reppold C. A busca de evidências de validade no desenvolvimento de instrumentos em Fonoaudiologia: revisão sistemática. Audiol Commun Res. 2015;20(4):371-83. http://dx.doi. org/10.1590/2317-6431-2015-1600.

24. Giusti E, Befi-Lopes D. Tradução e adaptação transcultural de instrumentos estrangeiros para o português brasileiro (PB). Pro Fono. 2008;20(3):207-10. http://dx.doi.org/10.1590/S0104-56872008000300012. PMid:18852970.

25. Nalom AF, Soares AJ, Cárnio MS. The relevance of receptive vocabulary in reading comprehension. CoDAS. 2015;27(4):333-8. http://dx.doi. org/10.1590/2317-1782/20152015016. PMid:26398255.

26. Kamhi AG, Clark M. Specific language impairment. 2013;111:219-27. http://dx.doi.org/10.1016/B978-0-444-52891-9.00022-1.

27. Kover ST, Weismer SE. Lexical characteristics of expressive vocabulary in toddlers with autism spectrum disorder. J Speech Lang Hear Res. 2014;57(4):1428-41. http://dx.doi.org/10.1044/2014_JSLHR-L-13-0006. PMid:24687027.

28. Kühn P, Sachse S, von Suchodoletz W. Language delay: what is the prognosis of late bloomers? Klin Padiatr. 2015;227(4):213-8. PMid:26038968

29. Pernambuco L, Espelt A, Magalhães HV, Lima KC. Recomendações para elaboração, tradução, adaptação transcultural e processo de validação de testes em Fonoaudiologia. CoDAS. 2017;29(3):e20160217. http:// dx.doi.org/10.1590/2317-1782/20172016217. PMid:28614460.

30. Cronbach LJ, Meehl PE. Construct validity in psychological tests. Psychol Bull. 1955;52(4):281-302. http://dx.doi.org/10.1037/h0040957. PMid:13245896. 